\title{
Práticas de gestão que influenciam o sucesso de novos produtos em empresas de base tecnológica
}

\author{
Daniel Jugend ${ }^{\mathrm{a}, *}$, Sérgio Luis da Silva ${ }^{\mathrm{b}}$ \\ a,* daniel@jugend.com.br, UFSCAR, Brasil \\ bsergiol@power.ufscar.br, UFCAR, Brasil
}

\begin{abstract}
Resumo
A excelência na gestão do processo de desenvolvimento de produtos (PDP) é fonte de vantagem competitiva para as empresas, especialmente para as de base tecnológica, que têm no lançamento de produtos com conteúdo tecnológico diferenciado importante fator de competitividade. 0 objetivo deste artigo é analisar práticas e fatores de sucesso relativos à gestão do PDP em empresas de base tecnológica de pequeno e médio porte do setor de automação de controle de processos. Para tanto, foram analisados fatores de gestão que influenciam o desempenho desse processo. 0 método de pesquisa adotado foi o survey, e os resultados enfatizam que o gerenciamento do PDP nas EBTs pesquisadas deve contemplar ações que valorizem as atividades de pré-desenvolvimento, tais como: a integração dos projetos de novos produtos com a estratégia da empresa, as características técnicas e econômicas que os produtos terão e o desenvolvimento das habilidades do gerente de projeto.
\end{abstract}

Palavras-chave

Processo de desenvolvimento de produtos. Empresas de base tecnológica. Setor de automação de controle de processos.

\section{Introdução}

As empresas de base tecnológica de menor porte têm no lançamento de produtos com alto conteúdo tecnológico importante fator de competitividade no mercado.

A possibilidade de manter um fluxo de produtos inovadores e competitivos depende da capacidade de desenvolvimento de tecnologias dessas empresas e da gestão do processo de desenvolvimento dos novos produtos e integração dessas novas tecnologias a esse processo. A capacidade de gerenciamento desse trabalho influi em fatores como tempo de lançamento do novo produto, inovação, custo e qualidade do produto desenvolvido.

Nos países em desenvolvimento, as pequenas e médias empresas de base tecnológica (EBTs) atuam essencialmente em nichos de mercado não ocupados pelas grandes empresas, e em substituição de importações (TOLEDO et al., 2008). Entretanto, não se deve desconsiderar o potencial econômico dessas empresas, afinal elas frequentemente

desenvolvem produtos inovadores e, dessa forma, podem impulsionar o crescimento econômico nas regiões onde atuam (YAP; SOUDER; BUISSON; GARRET, 1997), influenciando com uma cultura de inovação tecnológica os seus parceiros, clientes, fornecedores e concorrentes.

Grande parte das pesquisas de campo envolvendo EBTs de pequeno e médio porte no Brasil, segundo Fernandes, Côrtes e Oshi (2000), foca, prioritariamente, no desempenho dos polos tecnológicos e das incubadoras de empresas. Ainda de acordo com esses autores, há uma carência de pesquisas que revelem fatores gerencias críticos para o sucesso delas. E um processo crítico para essas empresas é o de desenvolvimento de produtos (DP), foco do presente estudo.

0 estudo da gestão do processo de desenvolvimento de produtos (PDP) em EBTs de pequeno e médio porte ainda encontra-se em fase incipiente no Brasil. De acordo com Maculan (2003), 
essas empresas enfrentam significativas dificuldades gerenciais, o que pode exercer influências sobre a taxa de sucesso de seus produtos.

Mesmo em países desenvolvidos há carência de estudos empíricos que revelem os fatores críticos de sucesso na gestão de desenvolvimento de produtos em EBTs de menor porte, conforme relatam os trabalhos de March-Chorda, Gunasekaran e Lloriaarambur (2002) e Souder, Buisson e Garret (1997).

As melhores práticas de gestão para pequenas e médias empresas somente podem ser recomendadas a partir da consideração de suas especificidades (LEONE, 1999). Daí a relevância de se identificar essas práticas em empresas de pequeno e médio porte em um setor industrial específico.

Levando-se em consideração o contexto apresentado, o objetivo desse artigo é caracterizar a gestão do PDP em EBTs de pequeno e médio porte do setor de automação de controle de processos (ACP) e também levantar e discutir fatores críticos de sucesso relativo à condução desse trabalho. Foram analisadas questões tais como o desempenho percebido do produto desenvolvido, o grau de inovação do produto, as habilidades da empresa e do líder de projeto, e qualidade de execução das atividades do PDP.

Essa pesquisa teve uma fase qualitativa e uma quantitativa, levantando-se informações e dados por meio da aplicação de questionários em visitas realizadas a 32 EBTs de pequeno e médio porte, do setor de ACP, localizadas no Estado de São Paulo. Essa região foi escolhida para a realização deste estudo por apresentar significativa concentração de EBTs e, de acordo com Fernandes, Côrtes e Oshi (2000), esse setor (ACP) é um dos mais representativos dentre as EBTs de menor porte desse estado.

As empresas do setor escolhido podem ser consideradas inovadoras, pois são classificadas como de média/alta intensidade de inovação tecnológica (de produto e processo) dentre os 33 setores pesquisados pelo Instituto Brasileiro de Geografia e Estatística - IBGE (2005), por meio da pesquisa nacional de inovação tecnológica (PINTEC). No estudo da PINTEC, o setor de ACP é definido como gerador e difusor de progresso técnico, com potencial para influenciar seus clientes e fornecedores.

Além disso, as empresas de ACP possuem certas características que trazem desafios adicionais para a gestão do PDP, como por exemplo: necessidade de domínio e transferência de diferentes tecnologias (óptica, eletrônica, mecatrônica, software, telemetria, entre outras) aplicadas aos seus produtos; e necessidade de forte integração com os clientes para a adequação do produto desenvolvido ao processo de produção da indústria-cliente.

0 artigo inicialmente apresenta uma breve revisão bibliográfica sobre gestão do PDP e seus fatores críticos de sucesso, em seguida conceitua EBTs do setor de ACP. Posteriormente, são apresentados o método empregado e discussões sobre caracterização e os fatores críticos de sucesso no PDP do setor estudado.

\section{Gestão do processo de desenvolvimento de produtos}

De maneira geral, desenvolver produtos segundo Rozenfeld et al. (2006) consiste em um conjunto de atividades por meio das quais busca-se, a partir das necessidades do mercado e possibilidades e restrições tecnológicas, e considerando as estratégias competitivas de uma empresa, chegar às especificações de um produto e de seu processo de produção.

Por situar-se na interface entre empresa e mercado, as atividades de desenvolvimento de produtos podem ser consideradas um dos principais processos de uma empresa e, para ser bem-sucedido, é relevante que as empresas empreguem boas práticas de gestão para conduzi-lo (ERNST, 2000; KAHN; BARCZAK; MOSS, 2006) . Com o intuito de indicar boas práticas para a gestão do PDP, diversos autores propõem modelos de etapas predefinidas para a sua condução (CLARK; WHEELWRIGHT, 1993; PUGH, 1996; ROZENFELD et al., 2006).

Dentre esses modelos, o proposto por Rozenfeld et al. (2006) é reconhecido como um trabalho recente, que contempla as boas práticas presentes em outros trabalhos clássicos sobre o tema gestão de desenvolvimento de novos produtos e ainda apresenta grande nível de detalhamento para as suas atividades (MIGUEL, 2008). Seguindo a obra de Rozenfeld, conforme ilustra a Figura 1, de maneira geral, as etapas de desenvolvimento de produto podem ser constituídas pelas seguintes macrofases: pré-desenvolvimento, desenvolvimento e pós-desenvolvimento.

Segundo Morgan e Liker (2008), visão de processo no desenvolvimento de produtos significa realizar todas suas atividades buscando atender às necessidades dos clientes desde a geração do conceito até a obsolescência do produto. A aplicação da abordagem por processos implica ainda, segundo Griffin e Hauser (1996), em uma integração harmônica entre as diversas funções que influenciam no desenvolvimento de produtos de uma empresa. 


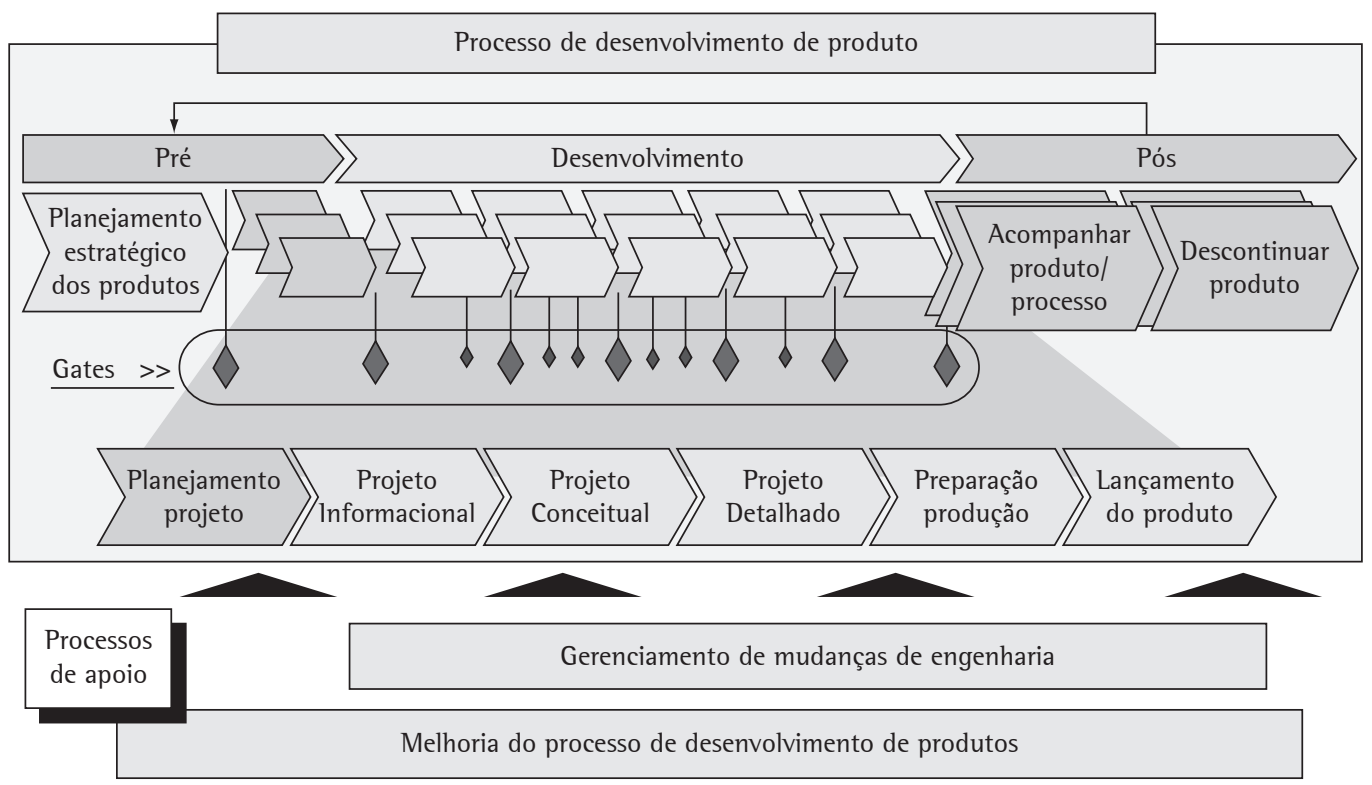

Figura 1. Visão geral do PDP. Fonte: Rozenfeld et al. (2006, p. 44).

O PDP de uma empresa é tido como um processo complexo, que para ser bem-sucedido exige adequada capacitação gerencial (CALANTONE; CHAN; CUl, 2006 ). Uma vertente de pesquisa sobre o tema gestão do PDP tem buscado compreender quais fatores podem ser compreendidos como críticos de sucesso associados a esse processo (YAP; SOUDER, 1994; SOUDER; BUISSON; GARRET; GRIFFIN, 1997; ERNST, 2002; KAHN; BARCZAK; MOSS, 2006; MOULTRIE; CLARKSON; PROBERT, 2007), isto é, fatores que podem levar um projeto de novo produto a ser bem ou malsucedido.

Baseado em publicações sobre esse tema, conforme demonstra o Quadro 1, esta pesquisa identificou determinados fatores que são críticos ao PDP. Esses fatores investigados são desdobrados em variáveis apresentadas em quadro presente no tópico de método de pesquisa, que explica a construção do questionário utilizado (item 4).

\section{Empresas de base tecnológica de pequeno e médio porte do setor de automação de controle de processos}

Segundo Fernandes, Côrtes e Oshi (2000) e Toledo et al. (2008), EBTs são organizações que têm no conhecimento um componente estratégico para a sua competitividade e realizam importantes esforços tecnológicos, uma vez que concentram grande parte de sua dedicação no desenvolvimento e fabricação de produtos que integram sistematicamente novas tecnologias.
Para o Sebrae/IPT (2001), as EBTs de menor porte são comprometidas com o projeto, desenvolvimento e produção de novos produtos e/ou processos, caracterizam-se pela aplicação sistemática do conhecimento técnico-científico, usam tecnologias inovadoras, têm alta proporção de gastos com pesquisa e desenvolvimento (P\&D), empregam grande porcentagem de pessoal técnico-científico e de engenharia e atendem mercados pequenos e específicos.

Diante dessas definições, esse artigo considera EBTs de pequeno e médio porte aquelas empresas que possuem basicamente as seguintes características: desenvolvem sistematicamente tecnologias e as transferem a um ou mais produtos durante o PDP e atendem a segmentos específicos (nichos) de mercado e/ou a substituição de importações.

Já as EBTs do setor de automação de controle de processos foram consideradas como aquelas empresas que fazem parte da indústria de automação industrial envolvidas com atividades de desenvolvimento e produção de produtos que visam o controle de processos de fabricação por meio da aplicação das tecnologias mecânica, eletrônica, telemetria e de informática (JUGEND; SILVA; TOLEDO, 2005).

Apresentada a revisão teórica sobre o tema e o setor pesquisado, o próximo tópico irá expor e discutir o método de pesquisa empregado nesse estudo.

\section{Método de pesquisa}

Para atingir o objetivo desse trabalho, tornou-se necessário, inicialmente, identificar a amostra a ser 
Quadro 1. Fatores críticos de sucesso para a gestão de desenvolvimento de novos produtos.

\begin{tabular}{|l|l|}
\hline \multicolumn{1}{|c|}{ Fator crítico de sucesso } & \multicolumn{1}{c|}{ Publicações } \\
\hline Grau de inovação do novo produto & $\begin{array}{l}\text { Garcia e Calantone (2002); Scott (2000); Calantone, Chan e Cui (2006); } \\
\text { Erhun, Gonçalves e Hopman (2007); Meyer (2008); Verganti (2008). }\end{array}$ \\
\hline Entendimento do mercado-alvo & $\begin{array}{l}\text { Souder, Buisson e Garret (1997); Koen et al. (2002); Bond e Houston (2003); } \\
\text { Griffin (2005). }\end{array}$ \\
\hline Características do produto & Clausing (1994); Mikkola (2001); Patterson (2005). \\
\hline Fontes de tecnologia & $\begin{array}{l}\text { Shulz et al. (2000); Kappel (2001); Drejer (2002); Bond e Houston (2003); } \\
\text { Meyer (2008). }\end{array}$ \\
\hline $\begin{array}{l}\text { Habilidades da empresa para } \\
\text { condução do PDP }\end{array}$ & $\begin{array}{l}\text { Griffin (1997); Olson et al. (2001); Koen et al. (2002); } \\
\text { Oliver, Dostaler e Dewberry (2004). }\end{array}$ \\
\hline Habilidades do líder do projeto & $\begin{array}{l}\text { Brown e Eisenhardt (1995); Griffin e Hauser (1996); Kim, Min e Cha (1999); } \\
\text { Koen et al. (2002); Thieme, Song e Shin (2003). }\end{array}$ \\
\hline Integração funcional & $\begin{array}{l}\text { Griffin e Hauser (1996); Drejer; Gunge e Holm (1997); Souder, Buisson e Garret (1997); } \\
\text { Song, Montoya-Weiss e Schmidt (1997); Creveling, Slutsky e Antis (2003); } \\
\text { Garcia, Sanzo e Trespalacios (2008). }\end{array}$ \\
\hline Organização da empresa para o PDP & Cusumano e Nobeoka (1998); Mendes e Toledo (2003); Koen (2005). \\
\hline $\begin{array}{l}\text { Qualidade da execução das atividades } \\
\text { do PDP }\end{array}$ & $\begin{array}{l}\text { Dooley, Subra e Anderson (2002); Carvalho (2001); Amaral, Rozenfeld e Araújo } \\
\text { (2007); Moultrie et al. (2007). }\end{array}$ \\
\hline
\end{tabular}

pesquisada. Conforme critérios do Sebrae (Serviço Brasileiro de Apoio às Micro e Pequenas Empresas) e do IBGE (Instituto Brasileiro de Geografia e Estatística), considerou-se como pequenas empresas aquelas com 20 a 99 empregados, e médias as com 100 a 499 empregados.

Como não foi encontrado, após extensas buscas, algum documento que listasse as EBTs de pequeno e médio porte do setor de ACP no estado de São Paulo, primeiramente foram enviados via correio eletrônico (emai) 350 questionários para todas as empresas de automação industrial, sediadas no estado e cadastradas no Congresso e Exposição Internacional de Automação (Conai), principal encontro de empresas desse setor. Nesse primeiro contato objetivou-se identificar quantas e quais se enquadravam como EBTs de pequeno e médio porte, e se elas podiam ser classificadas como de base tecnológica e se realizavam de maneira rotineira atividades de desenvolvimento de novos produtos.

Nesse primeiro contato obtiveram-se 52 respostas. Dentre essas empresas, 26 se enquadraram no perfil pretendido pela pesquisa. Visando ampliar a amostra, foram visitados sítios na internet de todas as empresas de automação industrial do estado de São Paulo, cadastradas na Associação Brasileira da Indústria Elétrica e Eletrônica (Abinee), além de outras empresas indicadas por profissionais da área de automação industrial. As que apontaram ser potenciais candidatas para participar desta pesquisa foram contatadas via telefone. Das empresas contatadas nesta segunda tentativa, 20 se enquadraram no perfil pretendido.
Chegou-se, portanto, a um conjunto identificado de 46 empresas, caracterizadas como EBTs de pequeno e médio porte do setor de ACP localizadas no estado. Todas foram contatadas via telefone para participar da pesquisa e, destas, 32 se dispuseram a ser visitadas para aplicação do questionário. Portanto, a amostra obtida representou aproximadamente $70 \%$ da população identificada.

Como essas empresas possuem características semelhantes, principalmente em termos de famílias de produtos que desenvolvem, tecnologias utilizadas e clientes atendidos, e também por atuarem em um mesmo setor econômico, pode-se considerar que essa é uma amostra representativa da população de EBTs de de pequeno e médio porte do setor de automação de controle de processos.

As entrevistas foram realizadas com os sóciosdirigentes, gerentes e engenheiros dessas empresas. Todos esses profissionais trabalham diretamente nas atividades de desenvolvimento de produtos.

Para operacionalizar o levantamento de dados foram utilizados dois questionários aplicados nessas entrevistas, os quais passaram por um pré-teste em duas empresas selecionadas, antes de tomarem a forma definitiva.

Com o objetivo de compreender práticas de gerenciamento do PDP, utilizou-se questionário semiestruturado qualitativo, que teve por objetivo levantar dados gerais e características das empresas, e também ter uma visão geral sobre a estruturação do seu PDP.

Após a aplicação desse questionário, visando identificar fatores que geram maior influência 
sobre o sucesso ou o não sucesso dos produtos desenvolvidos pelas empresas pesquisadas, utilizou-se um questionário estruturado quantitativo, que foi aplicado duas vezes em cada empresa. 0 primeiro preenchimento do questionário tinha como foco um projeto de desenvolvimento de produto considerado bem-sucedido pelo entrevistado, e o mesmo questionário era novamente aplicado focando em uma experiência de desenvolvimento vista como malsucedida. Os projetos deveriam ter sido conduzidos nos últimos cinco anos.

Seguindo o embasamento teórico apresentado no Quadro 1, esse questionário estruturado utilizou-se de uma escala likert que vai de 1 a 5 , sendo que o conteúdo dessas alternativas de resposta variava conforme o fator a ser respondido. 0 Quadro 2 apresenta esses fatores, variáveis e escalas.

A seguir são apresentados os resultados dessa pesquisa.

\section{Desenvolvimento de produtos: características e fatores críticos de sucesso}

0 tópico 5.1 apresenta os resultados referentes à caracterização das empresas e aspectos gerais para a gestão do PDP. 0 tópico 5.2 apresenta e discute os fatores críticos de sucesso para o PDP nas empresas pesquisadas.

\subsection{Caracterização das empresas e da gestão do processo de desenvolvimento de produtos}

Aproximadamente metade das empresas que foram pesquisadas (53\%) concentra-se na grande São Paulo (São Paulo e ABCD), enquanto a outra metade (47\%) encontra-se distribuída pelo interior do estado (São Carlos, região de Campinas, região de Marília e região de Ribeirão Preto).

Quanto ao tamanho, constatou-se que $84 \%$ das empresas são pequenas. Esse grande número de empresas de menor porte pode ser atribuído à prática comum de spin-off entre seus empreendedores. Observou-se durante a pesquisa que esses empreendedores adquiriram experiência e conhecimento em grandes empresas do setor e perceberam uma oportunidade de negócio de iniciar um pequeno empreendimento em determinado nicho de mercado que, muitas vezes, não era atendido pelas grandes empresas do setor.

Os principais setores atendidos por essas empresas são as seguintes indústrias: alimentícia; automotiva; de geração, transmissão e distribuição de energia; eletroeletrônica; química e petroquímica, além de outras de automação industrial.

A Tabela 1 apresenta as principais famílias de produtos desenvolvidos pelas empresas pesquisadas. Dentre esses produtos, verificou-se que $44 \%$ das empresas desenvolvem somente produtos de catálogo, outras $28 \%$ têm como estratégia só o desenvolvimento de produtos customizados (quando o cliente solicita determinado tipo de produto e, somente a partir de então, a empresa inicia o PDP). Os 28\% restantes são representados por empresas que desenvolvem tanto produtos de catálogos quanto sob encomenda.

Cerca de $60 \%$ das empresas possui procedimentos formalizados para a realização das atividades do PDP. Em 41\% do total de empresas essa formalização está associada à certificação ISO 9001. A importância dessa certificação decorre principalmente de exigências dos principais clientes, em geral representados por grandes indústrias, que passaram a pedi-la dos seus fornecedores de equipamentos de automação de controle de processos.

Quanto à inovação dos produtos que desenvolvem, observou-se que $81 \%$ das empresas utilizam-se de projetos do tipo plataforma, ou seja, desenvolvem um produto base e reutilizam suas informações e soluções em versões adaptadas, melhoradas ou estendidas de novos produtos (derivativos). Pela predominância dessa característica, projetos, pode-se afirmar que as empresas focam mais as inovações incrementais de produtos que as mudanças radicais.

Todas as empresas afirmaram prestar assistência técnica para os produtos que desenvolvem. A prestação de assistência técnica é tida como fator de vantagem competitiva para essas empresas, pois muitos de seus atuais clientes, que anteriormente importavam os equipamentos de automação de controle de processos, passaram a buscar fornecedores nacionais, visando, dessa forma, obter serviços de assistência técnica com menor custo e maior agilidade.

Constatou-se, conforme apresenta a Tabela 2, que o desenvolvimento interno de tecnologias (normalmente eletrônica, software, mecatrônica e de telemetria) constitui o principal mecanismo para o desenvolvimento das tecnologias que são transferidas aos produtos dessas empresas.

Durante as entrevistas, pôde-se perceber que essa predominância de esforços internos para obtenção de tecnologia ocorre, principalmente, em virtude do perfil do líder da função P\&D e/ou engenharia 
Quadro 2. Fatores, variáveis e escala utilizada para medir os fatores que influenciam o sucesso e o não sucesso do PDP.

Seção: Características do produto/mercado/fontes tecnológicas

\begin{tabular}{|c|c|c|}
\hline Fator & Variáveis & Escala \\
\hline $\begin{array}{c}\text { Características } \\
\text { do mercado-alvo }\end{array}$ & $\begin{array}{l}\text { - Havia forte sinergia entre os mercados já explorados pela empresa } \\
\text { e o mercado-alvo para esse novo produto. } \\
\text { - } 0 \text { mercado para esse tipo de produto encontrava-se em } \\
\text { crescimento, justificando o lançamento de um novo produto. } \\
\text { - A avaliação do potencial de mercado para esse projeto foi bem } \\
\text { realizada pela empresa. } \\
\text { - Os consumidores/clientes tinham grande desejo por esse tipo de } \\
\text { produto. } \\
\text { - Os requisitos dos usuários foram bem entendidos e traduzidos } \\
\text { corretamente para especificações do novo produto. }\end{array}$ & $\begin{array}{l}\text { Discordo totalmente (1) a } \\
\text { Concordo totalmente (5). }\end{array}$ \\
\hline $\begin{array}{l}\text { Características } \\
\text { do produto }\end{array}$ & $\begin{array}{l}\text { - } 0 \text { produto apresenta desempenho técnico superior aos } \\
\text { concorrentes. } \\
\text { - } 0 \text { produto oferece as mesmas soluções que os concorrentes, mas } \\
\text { com vantagem de menor preço. } \\
\text { - } 0 \text { produto apresenta praticamente as mesmas características dos } \\
\text { produtos dos concorrentes. } \\
\text { - } 0 \text { produto estava bem articulado com as estratégias competitivas } \\
\text { e de produto da empresa. }\end{array}$ & $\begin{array}{l}\text { Discordo totalmente (1) a } \\
\text { Concordo totalmente (5). }\end{array}$ \\
\hline $\begin{array}{l}\text { Fontes de } \\
\text { tecnologia }\end{array}$ & $\begin{array}{l}\text { - Contratação de pessoal externo para suprir competências não } \\
\text { existentes na empresa. } \\
\text { - Uso de estratégia de licenciamento. } \\
\text { - Alianças e parcerias com clientes. } \\
\text { - Alianças e parcerias com fornecedores. } \\
\text { - Alianças e parcerias com centros de pesquisa e universidades. } \\
\text { - Alianças e parcerias com outras entidades. } \\
\text { - Desenvolvimento próprio/interno. }\end{array}$ & $\begin{array}{l}\text { Muito fraca (1) } \\
\text { a Muito forte (5). }\end{array}$ \\
\hline \multicolumn{3}{|c|}{ Seção: Níveis de habilidade - Organização/empresa e líder do projeto } \\
\hline Fator & Variáveis & Escala \\
\hline $\begin{array}{l}\text { Habilidades da } \\
\text { empresa }\end{array}$ & $\begin{array}{l}\text { - No geral, a empresa tinha as habilidades técnicas necessárias à } \\
\text { execução do projeto. } \\
\text { - A área de P\&D/DP tinha a habilidade técnica necessária ao } \\
\text { projeto. } \\
\text { - A área comercial tinha a habilidade técnica necessária ao projeto. } \\
\text { - A área de manufatura tinha a habilidade técnica necessária ao } \\
\text { projeto. } \\
\text { - A área de assistência técnica tinha a habilidade técnica necessária } \\
\text { ao projeto. }\end{array}$ & $\begin{array}{l}\text { Discordo totalmente (1) a } \\
\text { Concordo totalmente (5). }\end{array}$ \\
\hline $\begin{array}{l}\text { Habilidades do } \\
\text { líder do projeto }\end{array}$ & $\begin{array}{l}\text { - } 0 \text { líder do projeto tinha a habilidade técnica necessária à condução } \\
\text { do projeto. } \\
\text { - } 0 \text { líder do projeto tinha a habilidade interpessoal/relacionamento } \\
\text { necessária à condução do projeto. } \\
\text { - } 0 \text { líder do projeto tinha a habilidade gerencial necessária à } \\
\text { condução do projeto. } \\
\text { - } 0 \text { líder do projeto conseguiu motivar as pessoas envolvidas com o } \\
\text { projeto. } \\
\text { - } 0 \text { líder do projeto tinha total autoridade para tomar decisões } \\
\text { relativas ao projeto. } \\
\text { - } 0 \text { estilo de liderança adotado pelo líder do projeto foi adequado } \\
\text { à sua execução, estimulando a comunicação e a gestão de } \\
\text { conflitos. } \\
\text { - } 0 \text { estilo de liderança possibilitou a participação dos membros do } \\
\text { time de desenvolvimento nas decisões do projeto. } \\
\text { - } 0 \text { pessoal do time de desenvolvimento estava motivado para a } \\
\text { execução desse projeto. }\end{array}$ & $\begin{array}{l}\text { Discordo totalmente (1) a } \\
\text { Concordo totalmente (5). }\end{array}$ \\
\hline
\end{tabular}


Quadro 2. Fatores, variáveis e escala utilizada para medir os fatores que influenciam o sucesso e o não sucesso do PDP.

\begin{tabular}{|c|c|c|}
\hline \multicolumn{3}{|c|}{ Seção: Características organizacionais do PDP } \\
\hline Fator & Variáveis & Escala \\
\hline lntegração & $\begin{array}{l}\text { - } 0 \text { envolvimento e suporte da alta administração foram decisivos } \\
\text { para a execução desse projeto. } \\
\text { - } 0 \text { projeto foi gerenciado articulado aos demais projetos em } \\
\text { andamento da empresa. } \\
\text { - No projeto houve adequado grau de integração entre comercial e } \\
\text { P\&D/DP. } \\
\text { - No projeto houve adequado grau de integração entre manufatura } \\
\text { e P\&D/DP. } \\
\text { - } 0 \text { projeto contou com participação de várias áreas/departamentos } \\
\text { na realização das atividades de geração e seleção de ideias. } \\
\text { - } 0 \text { projeto contou com participação de várias áreas/departamentos } \\
\text { na realização das atividades de análise de viabilidade. } \\
\text { - O projeto contou com participação de várias áreas/departamentos } \\
\text { na realização das atividades de desenvolvimento técnico (projeto } \\
\text { do produto). } \\
\text { - O projeto contou com participação de várias áreas/departamentos } \\
\text { na realização das atividades de construção de protótipos. } \\
\text { - O projeto contou com participação de várias áreas/departamentos } \\
\text { na realização das atividades de testes do produto/mercado. } \\
\text { - O projeto contou com participação de várias áreas/departamentos } \\
\text { na realização das atividades de lançamento comercial. }\end{array}$ & $\begin{array}{l}\text { Discordo totalmente (1) a } \\
\text { Concordo totalmente (5). }\end{array}$ \\
\hline Organização & $\begin{array}{l}\text { - Estrutura funcional } \\
\text { - Estrutura matricial } \\
\text { - Estrutura por projetos } \\
\text { Cada uma das estruturas foi explicada aos entrevistados }\end{array}$ & $\begin{array}{l}\text { Discordo totalmente (1) a } \\
\text { Concordo totalmente (5). }\end{array}$ \\
\hline \multicolumn{3}{|c|}{ Seção: Qualidade de execução das atividades do PDP } \\
\hline Fator & Variáveis & Escala \\
\hline $\begin{array}{l}\text { Qualidade de } \\
\text { execução de } \\
\text { atividades do } \\
\text { PDP }\end{array}$ & $\begin{array}{l}\text { - Atividades de geração e seleção de ideias. } \\
\text { - Atividades de análise de viabilidade (técnica e econômica). } \\
\text { - Atividades de desenvolvimento técnico (projeto do produto). } \\
\text { - Atividades de construção de protótipos. } \\
\text { - Atividades de realização de teste do produto/mercado. } \\
\text { - Atividades de lançamento comercial do novo produto. } \\
\text { - Atividades de preparação e acompanhamento de documentos e } \\
\text { relatórios necessários à homologação do produto. }\end{array}$ & $\begin{array}{l}\text { Péssima (1) a } \\
\text { Excelente (5). }\end{array}$ \\
\hline $\begin{array}{c}\text { Qualidade de } \\
\text { execução - } \\
\text { outras atividades }\end{array}$ & $\begin{array}{l}\text { - Fixação de metas e objetivos de desempenho para o projeto. } \\
\text { - Estabelecimento de pontos de decisão para as etapas do PDP, ou } \\
\text { seja, início de uma etapa posterior somente após a avaliação e } \\
\text { aprovação das atividades/resultados da etapa anterior. } \\
\text { - Produção de documentos (briefings, desenhos, resultados de testes } \\
\text { etc.) relativos à execução do projeto. } \\
\text { - Atendimento de normas legais necessárias ao produto. } \\
\text { - Grau de simultaneidade na realização das atividades do PDP. } \\
\text { - Ao final foi realizada uma avaliação geral para identificar os acertos } \\
\text { ou erros cometidos ao longo do projeto. }\end{array}$ & $\begin{array}{l}\text { Péssima (1) a } \\
\text { Excelente (5). }\end{array}$ \\
\hline
\end{tabular}

(normalmente seus próprios empreendedores). Esses profissionais exercem importantes papéis no que diz respeito ao desenvolvimento interno das tecnologias que são aplicadas nos produtos que as empresas desenvolvem.
No que se refere à parceria com outras empresas como forma de obtenção de tecnologias, foi possível observar que, em geral, ela ocorre com empresas que já são conhecidas pelo(s) proprietário(s)/dirigente(s) das empresas pesquisadas, e essas empresas 
Tabela 1.Principais famílias de produtos desenvolvidos.

\begin{tabular}{|c|c|}
\hline Família de produto & $\begin{array}{l}\text { (\%) de empresas que } \\
\text { desenvolvem* }\end{array}$ \\
\hline $\begin{array}{l}\text { Sistemas de controle } \\
\text { (temperatura, proximidade, } \\
\text { pressão, entre outros) }\end{array}$ & 25 \\
\hline Softwares voltados à automação & 22 \\
\hline $\begin{array}{l}\text { Indicadores (temperatura, } \\
\text { velocidade, potência, vazão, } \\
\text { entre outros) }\end{array}$ & 16 \\
\hline Painéis voltados à automação & 16 \\
\hline Sensores & 12 \\
\hline $\begin{array}{l}\text { Controladores lógico } \\
\text { programáveis (CLP) }\end{array}$ & 9 \\
\hline Conversores & 6 \\
\hline $\begin{array}{l}\text { Medidores (temperatura, energia, } \\
\text { tensão etc.) }\end{array}$ & 6 \\
\hline Monitores de temperatura & 6 \\
\hline \multicolumn{2}{|c|}{$\begin{array}{l}\text { *Todas as empresas pesquisadas desenvolvem mais de uma família de } \\
\text { produto. }\end{array}$} \\
\hline \multicolumn{2}{|c|}{$\begin{array}{l}\text { Tabela } 2 \text {. Fontes de obtenção de tecnologia no esforço total } \\
\text { de desenvolvimento de produto. }\end{array}$} \\
\hline Fontes de obtenção da tecnologia & (\%) de empresas \\
\hline Apenas desenvolvimento interno & 63 \\
\hline Parceria com outras empresas & 31 \\
\hline Apoio de consultores & 13 \\
\hline Parceria com universidades & 6 \\
\hline Parceria com centros de pesquisa & 3 \\
\hline
\end{tabular}

parceiras ficam responsáveis pelo desenvolvimento e aplicação de determinado tipo de tecnologia para os produtos que estão sendo desenvolvidos pelas empresas de automação de controle de processos.

\subsection{Fatores críticos de sucesso}

0 objetivo desse tópico é examinar fatores de mercado, gerenciais e técnicos que influenciaram projetos passados de desenvolvimento de produtos, considerados de sucesso e de não sucesso escolhidos dentre os realizados nos últimos 5 anos, nas 32 EBTs pesquisadas.

A amostra utilizada conta com 32 questionários preenchidos de projetos de desenvolvimento de produtos (DP) de sucesso e 23 de não sucesso. Nove empresas não apresentaram o caso específico de não sucesso, destas 7 justificaram que, por desenvolverem apenas projetos de DP customizados, não há possibilidade de ocorrer casos de não sucesso. Duas empresas não responderam a esse questionário por falta de tempo durante a entrevista e, mesmo se comprometendo a enviar o questionário via email, não o fizeram.
É preciso notar, também, que seguindo um modelo consagrado de pesquisa internacional com esse formato (YAP; SOUDER, 1994; SOUDER; BUISSON; GARRET, 1997), cada empresa definiu sucesso e não sucesso a partir de sua percepção (não se tentou padronizar esse aspecto).

A partir desses questionários preenchidos, inicialmente foi feita uma análise descritiva (distribuição conjunta) de cada variável individual (Quadro 2) contida no questionário estruturado, que teve por objetivo verificar a sua associação com o sucesso e não sucesso dos produtos desenvolvidos.

A análise dos dados foi utilizada associando as respostas das variáveis presentes nos questionários. Para verificar se respostas estavam associadas à escala proposta, utilizou-se o coeficiente de contingência (BUSSAD; MORETTIN, 2002).

Os cálculos necessários para auxiliar na análise foram realizados pelo software SPSS ${ }^{\circledR}$ (Statistical Package for the Social Sciences), utilizado para efetuar as análises descritivas (associação e correlação) e comparação de médias.

0 sucesso do projeto do produto foi analisado por meio de uma escala de 5 pontos, conforme demonstra o Quadro 2. É esperado que casos de não sucesso apresentem predominantemente a pontuação 1 ou 2 enquanto que produtos bem-sucedidos tenham uma pontuação de 3, 4 ou 5 nessas mesmas variáveis.

Para se analisar a associação das variáveis individuais com o sucesso ou não dos produtos desenvolvidos pelas empresas pesquisadas, utilizou-se, inicialmente, um coeficiente que indica o grau de confiança dessa associação (p-valor). As associações são consideradas significativas para valores de $p$ abaixo de 0,05 , ou seja $p \leq 5 \%$. Além do $p$-valor, empregou-se também o coeficiente de contingência que verifica se existe e quão forte é a associação entre a variável em questão com o sucesso e não sucesso dos produtos desenvolvidos. A Tabela 3 apresenta as variáveis com $p$-valores inferiores a 0,05 e os maiores coeficientes de contingência que foram obtidos nessa pesquisa.

Buscou-se também identificar os fatores críticos de sucesso, por meio de suas médias, para os projetos de sucesso e não sucesso. Aquelas que apresentaram maior amplitude das médias são as que devem merecer maior atenção gerencial no PDP. Ou seja, uma variável que obteve pontuação alta para os casos de sucesso e pontuação baixa para os casos de não sucesso pode ser considerada como crítica para que o produto desenvolvido seja considerado bem ou malsucedido. 
Tabela 3. Associação das variáveis estudadas com o sucesso ou não sucesso dos produtos desenvolvidos.

\begin{tabular}{|c|c|c|}
\hline Variáveis & p-valor & $\begin{array}{l}\text { Coeficiente } \\
\text { de } \\
\text { contingência }\end{array}$ \\
\hline Características do produto & - & - \\
\hline 0 produto oferece desempenho técnico superior aos concorrentes. & 0,001 & 0,509 \\
\hline 0 produto estava bem articulado com as estratégias competitivas e de produto da empresa. & 0,002 & 0,502 \\
\hline 0 produto oferece as mesmas soluções que os concorrentes, mas com vantagens de custos. & 0,004 & 0,482 \\
\hline Qualidade de execução das atividades do PDP & - & - \\
\hline $\begin{array}{l}\text { Atividades de preparação e acompanhamento de documentos e relatórios necessários à } \\
\text { homologação do produto. }\end{array}$ & 0,024 & 0,502 \\
\hline Atividades de análise de viabilidade (técnica e econômica). & 0,003 & 0,479 \\
\hline Habilidades do líder de projeto & - & - \\
\hline $\begin{array}{l}0 \text { estilo de liderança possibilitou a participação dos membros do time de desenvolvimento nas } \\
\text { decisões de projeto. }\end{array}$ & 0,009 & 0,444 \\
\hline 0 líder do projeto conseguiu motivar as pessoas envolvidas com o projeto. & 0,013 & 0,432 \\
\hline $\begin{array}{l}0 \text { estilo de liderança adotado pelo líder do projeto foi adequado à sua execução, estimulando a } \\
\text { comunicação e a gestão de conflitos. }\end{array}$ & 0,013 & 0,432 \\
\hline 0 pessoal do time de desenvolvimento estava motivado para a execução desse projeto. & 0,020 & 0,419 \\
\hline Características organizacionais - integração & - & - \\
\hline $\begin{array}{l}\text { No projeto houve adequado grau de integração entre comercial e P\&D/DP (time de } \\
\text { desenvolvimento). }\end{array}$ & 0,010 & 0,440 \\
\hline
\end{tabular}

Significante ao nivel $p \leq 0,05$.

A Tabela 4 apresenta as variáveis que tiveram maiores variabilidades (diferenças entre médias) de resposta para os casos de sucesso e não sucesso.

Ao examinar as Tabelas 3 e 4, observa-se que as variáveis que mais se apresentaram como críticas estão relacionadas à fase de pré-desenvolvimento. Momento em que a empresa deve buscar alinhar o portfólio de produtos à sua estratégia, definir conceitos gerais para os produtos e decidir quem serão os líderes de projetos, variáveis identificadas como críticas nessa pesquisa, conforme será discutido nos próximos parágrafos.

0 alinhamento entre os produtos a serem desenvolvidos com as estratégias competitivas de mercados da empresa foi apontado como fator crítico de sucesso, o qual deve estar presente desde a definição do portfólio de produtos a serem desenvolvidos e o conceito de cada um deles. Isso se torna evidente quando se analisa a variável: o produto estava bem articulado com as estratégias competitivas e de produto da empresa.

Esses resultados convergem com as propostas de Clark e Wheelwright (1993) e Cooper, Edgett e Kleinschmidt (2004), que apontam que um dos fatores fortemente associados ao não sucesso no desenvolvimento de produtos está associado à não articulação entre projetos de novos produtos e estratégia da empresa.

Nas Tabelas 3 e 4 pode ser observado que as variáveis que dizem respeito às vantagens em relação à concorrência (de custo e características técnicas do produto) também estão associadas ao seu sucesso. A realização dessas vantagens depende da definição de características que os produtos desenvolvidos vão ter (técnicas e econômicas), que ocorrem de forma detalhada nas fases de projeto do produto, o que também requer boa gestão na fase de pré-desenvolvimento.

Sobre a integração funcional, assim como os resultados das pesquisas de Souder, Buisson e Garret (1997), Ernst (2002) e Garcia, Sanzo e Trespalacios (2008), o presente estudo notou, conforme demonstra a Tabela 3, a importância da proximidade entre as áreas de desenvolvimento de produtos e comercial como fator determinante para o desenvolvimento de produtos e tecnologias viáveis comercialmente.

Além disso, a integração entre as áreas comercial e as responsáveis pelo desenvolvimento técnico de produtos é especialmente importante para as empresas de ACP, porque há necessidade de forte interação com seus clientes, na medida em que estes exigem adequada customização e/ou calibração do produto em conformidade com sua linha de produção.

Atividades de preparação e acompanhamento de documentos e relatórios necessários à homologação do produto foram variáveis apontadas como importantes pelas empresas. lsso pode ser justificado no setor de automação de controle de processos, devido às pressões impostas pelos clientes dessas empresas (manufatureiras), que, conforme explicado 
Tabela 4. Variáveis que apresentaram maior diferença entre médias das respostas que avaliam sucesso e não sucesso.

\begin{tabular}{|c|c|c|}
\hline Variáveis & $\begin{array}{c}\text { Média de respostas } \\
\text { para os casos de } \\
\text { sucesso }\end{array}$ & $\begin{array}{c}\text { Média de respostas } \\
\text { para os casos de } \\
\text { não sucesso }\end{array}$ \\
\hline Grau de inovação do produto & - & - \\
\hline 0 projeto resultou num produto derivativo & 3,41 & 2,13 \\
\hline Características do produto & - & - \\
\hline $\begin{array}{l}0 \text { produto estava bem articulado com as estratégias competitivas e de produto } \\
\text { da empresa. }\end{array}$ & 4,17 & 2,64 \\
\hline $\begin{array}{l}0 \text { produto oferece as mesmas soluções que os concorrentes, mas com vantagens } \\
\text { de custos. }\end{array}$ & 4,20 & 2,95 \\
\hline 0 produto oferece desempenho técnico superior aos concorrentes. & 4,06 & 2,90 \\
\hline Características do mercado -alvo & - & - \\
\hline $\begin{array}{l}\text { Havia forte sinergia entre os mercados já explorados pela empresa e o } \\
\text { mercado-alvo para esse novo produto. }\end{array}$ & 4,00 & 2,25 \\
\hline $\begin{array}{l}\text { A avaliação do potencial de mercado para esse projeto foi bem realizada pela } \\
\text { empresa. }\end{array}$ & 3,77 & 2,61 \\
\hline Qualidade de execução de atividades do PDP & - & - \\
\hline $\begin{array}{l}\text { Atividades de preparação e acompanhamento de documentos e relatórios } \\
\text { necessários à homologação do produto. }\end{array}$ & 4,35 & 3,00 \\
\hline
\end{tabular}

no tópico anterior, muitas vezes exigem o uso de procedimentos formalizados, que pode se dar, por exemplo, pela adoção da norma ISO 9000, para sistematizar as atividades de desenvolvimento de produtos e assegurar sua qualidade.

Com relação aos níveis de habilidade da empresa para a condução de projetos, verificou-se a importância do papel do líder de projeto, no que se refere, principalmente, à sua capacidade de motivar e possibilitar a participação dos diferentes especialistas envolvidos com projeto de desenvolvimento. Sobre esse fator, observou-se que tanto os produtos desenvolvidos de sucesso quanto os de não sucesso, normalmente, tinham um líder com competência técnica, ou seja, que possuía conhecimento na área técnica de produto e de processo. 0 diferencial nesse fator deu-se pela presença de competência gerencial do líder do projeto de desenvolvimento de produtos.

A capacidade gerencial desses líderes de projeto foi caracterizada, nessa pesquisa, pelas habilidades que este deve possuir em possibilitar a participação das diversas áreas funcionais da empresa, em motivar as pessoas envolvidas e liderar com a autoridade necessária todo o projeto de desenvolvimento de produto, o que demonstra que as empresas têm certa dependência em relação a seus líderes para as atividades de desenvolvimento de produtos.

Não se verificou correlação entre o sucesso dos produtos desenvolvidos com as fontes de tecnologias (tanto nos casos de sucesso quanto nos casos de não sucesso as variáveis relacionadas a esse fator obtiveram uma pontuação baixa). Essa pouca interação extrafirma para a obtenção ou desenvolvimento conjunto de tecnologias conduz a um questionamento sobre as considerações a respeito das EBTs de pequeno e médio porte, de que são empresas que possuem intensas relações de parceria com universidades e centros de pesquisa. Conforme demonstram os resultados observados na pesquisa atual, as empresas, em sua ampla maioria, não dependem e não se utilizam de relacionamento com universidades e centros de pesquisa para o desenvolvimento conjunto de tecnologias que são transferidas a seus produtos.

\section{Conclusões}

Em relação às práticas de gestão analisadas, verificou-se que as principais atividades que tornam os projetos de desenvolvimento de novos produtos bem ou malsucedidos são: a adequada articulação entre os projetos de produtos com a estratégia da empresa, uma análise mercadológica que leve em conta tanto as necessidades dos consumidores quanto o desempenho da concorrência e, também, as características que os produtos desenvolvidos terão (em termos de inovação e aspectos técnicos e econômicos).

Seguindo Rozenfeld et al. (2006), essas atividades fazem parte do pré-desenvolvimento, quando, tendo em vista os objetivos da empresa, são decididos os produtos que serão efetivamente desenvolvidos e incorporados ao seu portfólio.

As constatações desse trabalho, portanto, reforçam a necessidade do uso de boas práticas de gestão para macrofase de pré-desenvolvimento, ou seja, as fases de planejamento estratégico, revisão 
de portfólio e planejamento de projetos. 0 que inclui a adoção de práticas associadas ao PDP, tais como: adoção de equipe multifuncional, intensa integração entre as funções engenharia/P\&D e comercial/marketing e liderança que tenha ao mesmo tempo capacidade técnica e gerencial.

Porém, diferentemente do que aponta a literatura sobre EBTs, não se observou nessa pesquisa a adoção de relações de parcerias com outras instituições com o intuito de desenvolvimento conjunto de tecnologias para serem transferidas ao PDP.

Espera-se que os resultados deste trabalho possam somar-se ao corpo teórico sobre gestão do desenvolvimento de produtos e, ao mesmo tempo, contribuir para melhorias em indicadores de desempenho de projetos ao evidenciar práticas que condicionam o sucesso de um novo produto e que, portanto, merecem atenção de gestores envolvidos com o PDP.

\section{Referências}

AMARAL, D. C.; ROZENFELD, H.; ARAUJO, C. A case study about product development process evaluation. In: LOUREIRO, G. C.; CURRAN, R. (Eds.). Complex systems concurrent engineering: collaboration, technology innovation and sustainability. London: Springer-Verlag, 2007.

BOND, E. U.; HOUSTON, M. B. Barriers to matching new technologies and market opportunities in established firms. The Journal of Product Innovation Management, v. 20, n. 2, p. 120-135, 2003.

BROWN, S. L.; EISENHARDT, K. M. Product development - past research, present findings, and future-directions. Academy of Management Review, v. 20, n. 2, p. 343-378, 1995.

BUSSAD, W.; MORETTIN, P. Estatística básica. 5. ed. São Paulo: Saraiva, 2002.

CALANTONE, R. J.; CHAN, K.; CUI, A. S. Decomposing product innovativeness and its effects on new product success. The Journal of Product Innovation Management, v. 23, n. 5, p. 408-421, 2006.

CARVALHO, M. M. Qualidade em projeto. In: AMATO NETO, J. (Org.). Manufatura de classe mundial: conceitos, estratégias, aplicações. São Paulo: Atlas, 2001. p. 130-145.

CLARK, K. B.; WHEELWRIGHT, S. C. Managing new product and process development: text and cases. New York: The Free Press, 1993.

CLAUSING, D. Total quality development: a step-by-step guide to world-class concurrent engineering. New York: Asme, 1994.

COOPER, R. G.; EDGETT, S. J.; KLEINSCHMIDT, E. K. Benchmarking best NPD practices-1: culture, climate, teams and senior management's roles are the focus of this first in a 3-parts series. Research Technology Management, v. 47, n. 1, p. 31-43, 2004.

CREVELING, C. M.; SLUTSKY, J. L.; ANTIS, D. Design for six sigma: in technology \& product development. New Jersey: Pretince Hall, 2003.

CUSUMANO, M. A.; NOBEOKA, K. Thinking beyond lean: how multiple projects is transforming product development at Toyota and others companies. New York: The Free Press, 1998.

DOOLEY, K.; SUBRA, A.; ANDERSON, J. Adoption rates and patterns of best practices in new product development.
International Journal of Innovation Management, v. 6, n. 1, p. 85-103, 2002.

DREJER, A. Integrating product and technology development. International Journal Technology Management, v. 24 n. 2-3, p. 124-142, 2002

DREJER, A.; GUNGE, S.; HOLM, G. Management of technology in a complex world. International Journal of Materials and Product Technology, v. 12, n. 6-4, p. 239-254, 1997.

ERHUN, F.; GONÇALVES, P.; HOPMAN, J. The art of managing new products transitions. MIT Sloan Management Review, v. 48 , n. 3, p. 73-80, 2007.

ERNST, $H$. Success factors of new products development: a review of the empirical literature. International Journal of Management Reviews, v. 4, n. 1, p. 1-40, 2002.

FERNANDES, A. C.; CÔRTES, M. R.; OSHI, J. Innovation Characteristics of Small and Medium Sized TechnologyBased Firms in São Paulo, Brazil: a preliminary analysis. In: PROCEEDINGS OF INTERNATIONAL CONFERENCE OF TECHNOLOGY POLICY AND INNOVATION, 4., 2000, Curitiba. Anais..

GARCIA, R.; CALANTONE, R. A critical look at technological innovation typology and innovativeness terminology: a literature review. Journal of Product Innovation Management. v. 19, n. 2, p. 110-132, 2002.

GARCIA, R.; SANZO, M. J.; TRESPALACIOS, J. A. New product internal performance and market performance: evidence from Spanish firms regarding the role of trust, interfunctional integration, and innovation type. Technovation, v. 28, n. 11, p. 713-725, 2008.

GRIFFIN, A. Obtaining customer needs for product development. In: KAHN, K. B. (Ed.). The PDMA handbook of new product development. 2 ed. New Jersey: John Wiley \& Sons, 2005. p. 211-227.

GRIFFIN, A. PDMA Research on new product development practices: updating trends and benchmarking best practices. Journal of Product Innovation Management, v. 14, n. 6 , p. 429-459, 1997.

GRIFFIN, A.; HAUSER, J. R. Integrating R\&D and marketing: a review and analysis of the literature. Journal of Product Innovation Management, v. 13, n. 3, p. 191-215, 1996.

INSTITUTO BRASILEIRO DE GEOGRAFIA - IBGE. Pesquisa Industrial de Inovação Tecnológica: 2003. Rio de Janeiro, 2005.

JUGEND, D.; SILVA, S. L.; TOLEDO, J. C. Esforco inovador presente em empresas de base tecnológica de pequeno e médio porte: a perspectiva do setor de automação industrial. In: SEMINÁRIO LATINO-IBEROAMERICANO DE GESTIÓN TECNOLOGICA (ALTEC): INNOVACIÓN TECNOLÓGICA, COOPERACIÓN Y DESARROLLO, 11., 2005, Salvador. Anais..

KAHN, K. B.; BARCZAK, G.; MOSS, R. Perspective: establishing an NPD best practices framework. The Journal of product innovation management, v. 23, n. 2, p. 106-116, 2006.

KAPPEL, T. A. Perspectives on roadmaps: how organisations talk about the future. The Journal of product innovation management, v. 18, n. 1, p. 39-50, 2001.

KIM, Y.; MIN, B.; CHA, J. The roles of R\&D team leaders in Korea: a contingent approach. $R \& D$ Management, v. 29, n. 2, p. 153-165, 1999.

KOEN, P. A. et al. Fuzzy front end: effective methods, tools, and techniques. In: BELLIVEAN, P.; GRIFFIN, A.; SOMERMEYER, S. (Eds.). The PDMA Handbook of new product development. New Jersey: John Wiley \& Sons, 2002. p. 1-35.

KOEN, P. A. Innovation in large companies: approaches and organizational architeture. In: KAHN, K. B. (Ed.). The PDMA Handbook of new product development. 2 ed. New Jersey: John Wiley \& Sons, 2005. p. 111-126.

LEONE, N. M. C. P. G. As especificidades das pequenas e médias empresas. Revista de Administração, v. 34, n. 2, p. 91-94, 1999. 
MACULAN, A. M. Ambiente empreendedor e aprendizado das pequenas empresas de base tecnológica. In: LASTRES, H. M. M.; CASSIOLATO, J. E.; MACIEL, M. L. Pequena empresa: cooperação e desenvolvimento local. Rio de Janeiro: Relume Dumará: UFRJ, 2003. p. 311-327.

MARCH-CHORDȦ, 1.; GUNASEKARAN, A.; LLORIA-ARAMBURO, B. Product development process in Spanish SMEs: an empirical research. Technovation, v. 22, n. 5, p. 301-312, 2002.

MENDES, G. H. S.; TOLEDO, J. C. Uma visão dos principais arranjos organizacionais aplicados ao desenvolvimento de produto. In: CONGRESSO BRASILEIRO DE GESTÃO DO DESENVOLVIMENTO DE PRODUTOS, 4., 2003, Gramado. Anais...

MEYER, M. H. Perspective: how Honda innovates. The Journal of Product Innovation Management, v. 25, n. 3, p. 261-271, 2008.

MIGUEL, P. A. C. Implementação da gestão de portfólio de novos produtos: um estudo de caso. Revista Produção, v. 18 , n. 2 , p. $388-404,2008$.

MIKKOLA, J. M. Portfolio management of R\&D projects: implications for innovation management. Technovation, v. 21, n. 7, p. 423-435, 2001.

MORGAN, J. M.; LIKER, J. K. Sistema Toyota de desenvolvimento de produtos: integrando pessoas, processos e tecnologia. Porto Alegre: Bookman, 2008

MOULTRIE, J. P.; CLARKSON, J.; PROBERT, D. Development of a design audit tool for SME. The Journal of Product Innovation Management, v. 24, n. 4, p. 335-368, 2007.

OLIVER, N.; DOSTALER, I.; DEWBERRY, E. New product development benchmarks: the Japanese, North American, and UK consumer electronics industries. The Journal of High Technology Management Research, v. 15, n. 2, p. 249-265, 2004.

OLSON, E. M. et al. Patterns of cooperation during new product development among marketing, operations and R\&D: implications for project performance. Journal of Product Innovation Management, v. 18, p. 258-271, 2001.

PATTERSON, M. L. New product portfólio planning and management. In: KAHN, K. B. (Ed.). The PDMA handbook of new product development. 2. ed. New Jersey: John Wiley \& Sons, 2005. p. 46-58.
PUGH, S. Creating innovative products using total design: the living legacy of Stuart Pugh. Massachusetts: AddisonWesley, 1996.

ROZENFELD, H. et al. Gestão de desenvolvimento de produto: uma referência para a melhoria do processo. São Paulo: Saraiva, 2006

SCOTT, G. M. Critical technology management issues of new product development in high-tech companies. Journal of Product Innovation Management, v. 17, n. 2, p. 57-77, 2000

SERVICO DE APOIO ÀS MICRO E PEQUENAS EMPRESAS DE SÃO PAULO - SEBRAE; INSTITUTO DE PESQUISAS TECNOLÓGICAS - IPT. MPES de base tecnológica: conceituação, formas de financiamento e análise de casos brasileiros. São Paulo, 2001. (Relatório de pesquisa)

SHULZ, A. P. et al. Development and integration of winning technologies as key to competitive advantage. Systems Engineering, v. 3, n. 4, p. 180- 211, 2000.

SONG, X. M.; MONTOYA-WEISS, M. M.; SCHMIDT, J. B. Antecedents and consequences of cross-functional cooperation: a comparison of $\mathrm{R} \& \mathrm{D}$, manufacturing and marketing perspectives. Journal of Product Innovation Management, v. 47, n. 1, p. 14-35, 1997.

SOUDER, W. E.; BUISSON, D.; GARRET, T. Sucess throught customer-driven new product development: a comparison of US and New Zealand small entrepreneurial hight tecnology firms. Journal of Product Innovation Management, v. 14, n. 6, p. 459-472, 1997.

THIEME, R. J.; SONG, M. X.; SHIN, G. Project Management characteristics and new product survival. Journal of Product Innovation Management, v. 20, n. 2, p. 114-119, 2003.

TOLEDO, J. C. et al. Fatores críticos de sucesso no gerenciamento de projetos de desenvolvimento de produto em empresas de base tecnológica de pequeno e médio porte. Gestão \& Produção, v. 15, n. 1, p. 117-134, 2008.

VERGANTI, R. Design, meanings and radical innovation: a metamodel and research agenda. Journal of Product Innovation Management, v. 25, n. 5, p. 436-456, 2008.

YAP, C. M.; SOUDER, W. E. Factors influencing new product success and failure in small entrepreneurial hightechnology electronics firms. Journal of Product Innovation Management, v. 11, n. 5, p. 418-432, 1994.

\title{
Management practices influencing new product success in technology-based companies
}

\begin{abstract}
Proficiency in management activities undertaken in product development processes is regarded as a key competitive advantage for companies, particularly for small and medium sized technology-based firms that have the offering of high-tech products as their main market differential factor. The present study aims to promote an analysis of the practices carried out in the management of product development processes of small and medium sized technologybased companies of process control automation. The data was obtained through a survey, among the main results observed, it was verified that the management techniques incorporated in the project and pre-development phases, especially those implemented to obtain the product characteristics, the synchrony between the company strategy and projects, technical and economic characteristics those products will have, and the development of the project manager managerial skills.
\end{abstract}

\section{Keywords}

Management product development process. Technology-based companies. Process control automation. 